\title{
Pre-discovery Activity of New Interstellar Comet 2I/Borisov beyond 5 au
}

\author{
Quanzhi Ye (叶泉志) $)^{1}$ (D) Michael S. P. Kelley ${ }^{1}$ (1) , Bryce T. Bolin ${ }^{2}$, Dennis Bodewits ${ }^{3}$ (), Davide Farnocchia ${ }^{4}$ (1), \\ Frank J. Masci ${ }^{2}$ (1) , Karen J. Meech ${ }^{5}$ (1), Marco Micheli ${ }^{6,7}$ (1), Robert Weryk ${ }^{5}$ (10), Eric C. Bellm ${ }^{8}$ (10), Eric Christensen ${ }^{9}$, \\ Richard Dekany ${ }^{10}$, Alexandre Delacroix ${ }^{10}$, Matthew J. Graham ${ }^{11}$ (1) , Shrinivas R. Kulkarni ${ }^{11}$ (i), Russ R. Laher $^{2}{ }^{(0)}$, \\ Ben Rusholme ${ }^{2}$ (i), and Roger M. Smith ${ }^{10}$ \\ ${ }_{1}^{1}$ Department of Astronomy, University of Maryland, College Park, MD 20742, USA; qye@umd.edu \\ ${ }^{2}$ IPAC, California Institute of Technology, 1200 E. California Boulevard, Pasadena, CA 91125, USA \\ ${ }^{3}$ Physics Department, Leach Science Center, Auburn University, Auburn, AL 36849, USA \\ ${ }^{4}$ Jet Propulsion Laboratory, California Institute of Technology, 4800 Oak Grove Drive, Pasadena, CA 91109, USA \\ ${ }^{5}$ Institute for Astronomy, University of Hawaii, 2680 Woodlawn Drive, Honolulu, HI 96822, USA \\ ${ }^{6}$ ESA NEO Coordination Centre, Largo Galileo Galilei, 1, I-00044 Frascati (RM), Italy \\ ${ }^{7}$ INAF-Osservatorio Astronomico di Roma, Via Frascati, 33, I-00040 Monte Porzio Catone (RM), Italy \\ 8 DIRAC Institute, Department of Astronomy, University of Washington, 3910 15th Avenue NE, Seattle, WA 98195, USA \\ ${ }^{9}$ University of Arizona, Lunar and Planetary Laboratory, 1629 E. University Boulevard, Tucson, AZ 85721, USA \\ ${ }^{10}$ Caltech Optical Observatories, California Institute of Technology, Pasadena, CA 91125, USA \\ ${ }^{11}$ Division of Physics, Mathematics, and Astronomy, California Institute of Technology, Pasadena, CA 91125, USA \\ Received 2019 November 6; revised 2019 December 5; accepted 2019 December 23; published 2020 January 31
}

\begin{abstract}
Comet 2I/Borisov, the first unambiguous interstellar comet ever found, was discovered in 2019 August at $\sim 3$ au from the Sun on its inbound leg. No pre-discovery detection beyond 3 au has yet been reported, mostly due to the comet's proximity to the Sun as seen from the Earth. Here we present a search for pre-discovery detections of comet Borisov using images taken by the Catalina Sky Survey, Pan-STARRS, and the Zwicky Transient Facility (ZTF), with a further comprehensive follow-up campaign being presented in Bolin et al. We identified comet Borisov in ZTF images taken in 2019 May and use these data to update its orbit. This allowed us to identify the comet in images acquired as far back as 2018 December, when it was 7.8 au from the Sun. The comet was not detected in 2018 November when it was 8.6 au from the Sun, possibly implying an onset of activity around this time. This suggests that the activity of the comet is either driven by a more volatile species other than $\mathrm{H}_{2} \mathrm{O}$, such as $\mathrm{CO}$ or $\mathrm{CO}_{2}$, or by exothermic crystallization of amorphous ice. We derive the radius of the nucleus to be $<7 \mathrm{~km}$ using the non-detection in 2018 November, and estimate an area of $\sim 0.5-10 \mathrm{~km}^{2}$ has been active between 2018 December and 2019 September, though this number is model-dependent and is highly uncertain. The behavior of comet Borisov during its inbound leg is observationally consistent with dynamically new comets observed in our solar system, suggesting some similarities between the two.
\end{abstract}

Unified Astronomy Thesaurus concepts: Comets (280); Small solar system bodies (1469); Solar system astronomy (1529)

\section{Introduction}

Comet 2I/Borisov ${ }^{12}$ (2I for short), discovered by G. Borisov at the Crimean Astrophysical Observatory on 2019 August 30, is the second interstellar object and the first unambiguous interstellar comet ever found. Early observations of 2I have revealed a sizable coma (de León et al. 2019; Guzik et al. 2019; Jewitt \& Luu 2019) and the detection of the emission from CN and atomic oxygen (Fitzsimmons et al. 2019; McKay et al. 2020; Opitom et al. 2019). This makes it distinctively different from the first discovered interstellar object 1I/'Oumuamua, which did not exhibit a detectable cometary feature such as a coma and/or a tail (Meech et al. 2017; Ye et al. 2017; Oumuamua ISSI Team et al. 2019). The detection of activity is important, as it provides a way to probe the composition of the nucleus, which can be done through a direct analysis of gases in the coma, and by observing how the activity responds to different levels of insolation at different heliocentric distances.

2I was discovered at a heliocentric distance $r_{\mathrm{H}}=2.98 \mathrm{au}$, with a brightness $(V=18 \mathrm{mag}$ ), which is well within the reach of modern near-Earth object (NEO) surveys. The belated discovery

\footnotetext{
${ }_{12}$ Formerly designated C/2019 Q4 (Borisov). See Minor Planet Electronic Circular (MPEC) 2019-R106 and MPEC 2019-S72.
}

is primarily due to the fact that the comet was within $45^{\circ}$ from the Sun, within the typical solar avoidance zone for ground-based optical telescopes, from 2019 early May to early September.

A detection (or non-detection) at $r_{\mathrm{H}} \gg 3$ au would better constrain the size of the nucleus (as the comet is likely less active) as well as its inbound orbit, and would be diagnostic of the comet's volatile composition (Fitzsimmons et al. 2019), since the activity of most known (i.e., solar system) comets within $\sim 3-5$ au is driven by water ice sublimation (e.g., Meech \& Svoren 2004). All-sky, time-domain surveys allow for serendipitous observations of objects before they are discovered. Here we present a search for pre-discovery detections of 2I in the data of several sky surveys. We will describe the search process in Section 2, the procedure of photometric analysis in Section 3, and discuss the implications of the detections and non-detections in Sections 4 and 5.

\section{Pre-discovery Detections}

\subsection{Zwicky Transient Facility (ZTF)}

The ZTF is a wide-field optical survey utilizing the $1.2 \mathrm{~m}$ Palomar Oschin Schmidt and a dedicated camera with a $55 \mathrm{deg}^{2}$ field of view (Bellm et al. 2019; Graham et al. 2019; 
Table 1

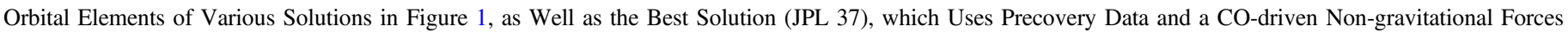

\begin{tabular}{|c|c|c|c|c|}
\hline Parameter & $\begin{array}{c}\text { Gravity-only } \\
\text { (without precovery) }\end{array}$ & $\begin{array}{c}\text { Nongrav- } \mathrm{H}_{2} \mathrm{O} \\
\text { (without precovery) }\end{array}$ & $\begin{array}{c}\text { Nongrav-CO } \\
\text { (without precovery) }\end{array}$ & $\begin{array}{c}\text { Final-CO } \\
\text { (with precovery) }\end{array}$ \\
\hline Epoch (TDB) & 2019 Sep 16.0 & 2019 Sep 16.0 & 2019 Sep 16.0 & 2019 Dec 20.0 \\
\hline Perihelion time (TDB) & 2019 Dec $8.65 \pm 0.07 \mathrm{~d}$ & 2019 Dec $13 \pm 1$ day & 2019 Dec $9.1 \pm 0.4$ day & 2019 Dec $8.551 \pm 0.001$ day \\
\hline Perihelion distance $q(\mathrm{au})$ & $2.003 \pm 0.003$ & $1.85 \pm 0.05$ & $1.98 \pm 0.02$ & $2.00664 \pm 0.00004$ \\
\hline Eccentricity $e$ & $3.34 \pm 0.01$ & $2.7 \pm 0.2$ & $3.27 \pm 0.07$ & $3.3576 \pm 0.0003$ \\
\hline Inclination $i$ & $44^{\circ} .08 \pm 0.03$ & $45^{\circ} 6 \pm 0.5$ & $44.3 \pm 0.2$ & $44^{\circ} .0515 \pm 0.0004$ \\
\hline Long. Ascending Node $\Omega$ & $308.12 \pm 0.03$ & $306.7 \pm 0.4$ & $308^{\circ} 0 \pm 0.2$ & $308.1488 \pm 0.0004$ \\
\hline Argument of Perihelion $\omega$ & $209^{\circ} .21 \pm 0.06$ & $213^{\circ} \pm 1^{\circ}$ & $209.7 \pm 0.5$ & $209^{\circ} .1227 \pm 0.0009$ \\
\hline Radial accel. $A_{1}\left(\right.$ au day $\left.^{-2}\right)$ & $\ldots$ & $(3.5 \pm 0.7) \times 10^{-4}$ & $(1.4 \pm 1.5) \times 10^{-5}$ & $(-4.4 \pm 3.2) \times 10^{-8}$ \\
\hline Transverse accel. $A_{2}\left(\right.$ au day $\left.{ }^{-2}\right)$ & $\cdots$ & $(-2.7 \pm 0.3) \times 10^{-4}$ & $(-6.2 \pm 5.6) \times 10^{-6}$ & $(1.3 \pm 0.3) \times 10^{-7}$ \\
\hline
\end{tabular}

Masci et al. 2019). Apart from two baseline surveys and a number of mini-surveys, ZTF executes a mini-survey ("Twilight Survey") that observes regions with solar elongation down to $35^{\circ}$ (Ye et al. 2020), which is particularly suitable for the search of pre-discovery detections of 2I. The first phase of the ZTF Twilight Survey was executed from 2018 November to 2019 June, fortuitously covering the period before 2I reached solar conjunction in 2019 July.

Using the ZChecker comet monitoring package (Kelley et al. 2019) and JPL orbit solution \#12 (the most recent JPL solution at the time that the search was conducted), we identified a total of 202 images from 2018 October 1 to the discovery date of 2I (2019 August 30) that potentially contained the comet. All images use $30 \mathrm{~s}$ exposure times, and were taken in a wide range of observing circumstances, with $5 \sigma$ limiting magnitudes between 16 and 21 and seeing between $1^{\prime \prime}$ and $5^{\prime \prime}$. The comet reached solar conjunction in 2019 July, and the last three sets of images acquired before that were taken in the course of the Twilight Survey on 2019 April 29, May 2, and 5 respectively, when 2I was at $r_{\mathrm{H}}=5.20-5.09$ au. Prior to these Twilight Survey data, the last image was taken on 2019 April 16, when the comet was at $r_{\mathrm{H}}=5$. $45 \mathrm{au}$. The April 29 and May 5 observations were not optimal because only a few images were acquired, there was a high sky background, and/or the comet was near image edges. The May 2 observations were particularly fortuitous as the comet was located in an overlapping strip between two fields, and thus had $2 \times$ more images than we typically have (eight images total, instead of the typical four images per field). Therefore, we focused on the 2019 May 2 data for our initial search of a pre-discovery detection.

The astrometric observations available up to this point did not provide meaningful constraints on the cometary non-gravitational perturbations (e.g., Yeomans et al. 2004), which lead to different orbit solutions. To capture the ephemeris variations using different model assumptions, we fit the available astrometry as of 2019 October 3 using a gravity-only model, and two nongravitational models that either assumed sublimation of $\mathrm{H}_{2} \mathrm{O}$ (Marsden et al. 1973) or CO (with sublimation rate $\left.\propto r_{\mathrm{H}}^{-2}\right)^{13}$ to be the primary driver of comet activity. We then combined all eight images from May 2 following the apparent motion of 2I. The three solutions are tabulated in Table 1 (together with the final

\footnotetext{
13 To our best knowledge, the only CO-driven non-gravitational model published to-date is the Yabushita (1996) model. However, their elbow at $\sim 5$ au is inconsistent with both theoretical and observational results, which suggests an elbow of $\gg 10$ au (e.g., Biver et al. 1996; Gunnarsson et al. 2002; Meech \& Svoren 2004, M.- T. Hui et al. 2020, in preparation). Here we simply assume the sublimation rate follows $\propto r_{\mathrm{H}}^{-2}$, since the elbow is likely much larger than the largest $r_{\mathrm{H}}$ in our data set.
}

orbit, after we have successfully identified the comet in the ZTF pre-discovery data, as we discuss below), and the combined image as well as the uncertainty ellipses of the three solutions are shown as Figure 1. Because of the short arc and the low elongation of many of the astrometric positions, we note that the estimated non-gravitational parameters could be unreliable. In particular, the parameters for the $\mathrm{H}_{2} \mathrm{O}$-driven model appear to be too large to be credible, and are possibly caused by astrometric biases at such a small solar elongation.

We identified a possible detection at a signal-to-noise ratio of $\sim 10$ on the combined May 2 image (Figure 2). The detection is within the $3 \sigma$ uncertainty ellipses of gravity-only and $\mathrm{CO}$ nongravitational solutions, and about $5^{\prime \prime}$ southeast of the predicted Minor Planet Center (MPC) position. Visually, the object is about $\sim 5^{\prime \prime}$ in diameter and is largely circular in shape, with no apparent sign of a tail. The object is barely visible on individual frames (Figure 2), with a motion consistent with that of 2I.

We then examined other images in the time period in question, using the same shift-and-stack technique as outlined above. The detection on 2019 May 2, if real, would have greatly reduced the ephemeris uncertainty from a few arcminutes to a few arcseconds back to 2019 January, and would enable more pre-discovery data to be found. Since the comet is extremely faint, in order to eliminate contamination due to variable sky conditions and passing background stars, we only use frames that have (1) a $5 \sigma$ limit of $r \gtrsim 19.5$, (2) an average full-width-half-maximum (FWHM) of $<3$ pixels, and (3) no background stars that are within $10^{\prime \prime}$ from the predicted position of the comet.

All the pre-discovery detections and non-detections are summarized in Table 2. We were able to trace the object back to 2018 December 13. Apart from the 2019 May 2 data, the object is not visible in individual frames, and a clear detection usually requires stacking images from multiple nights. By including these astrometric positions ${ }^{14}$ with the post-discovery astrometric measurements of $2 \mathrm{I}$ and considering the nongravitational effect, we were able to achieve a satisfactory orbital fit with residuals of order $1^{\prime \prime}$, which is slightly above the average compared to typical ZTF astrometry (better than 0 ".5). This is due to a weak systematic bias in the data, which is possibly caused by the fact that most astrometric data were taken at low solar elongation and therefore at high airmass, introducing some differential color refraction bias (see also the discussion in Section 4.4). Nevertheless, we identify the object observed from 2018 December 13 to 2019 May 5 as 2I.

A point worth addressing is the non-detection in November 2018. The $3 \sigma$ uncertainty of the position is $\sim 4^{\prime \prime}$ ( 4 pixels)

$\overline{14}$ Published in MPEC 2019-V34 and MPEC 2019-W50. 


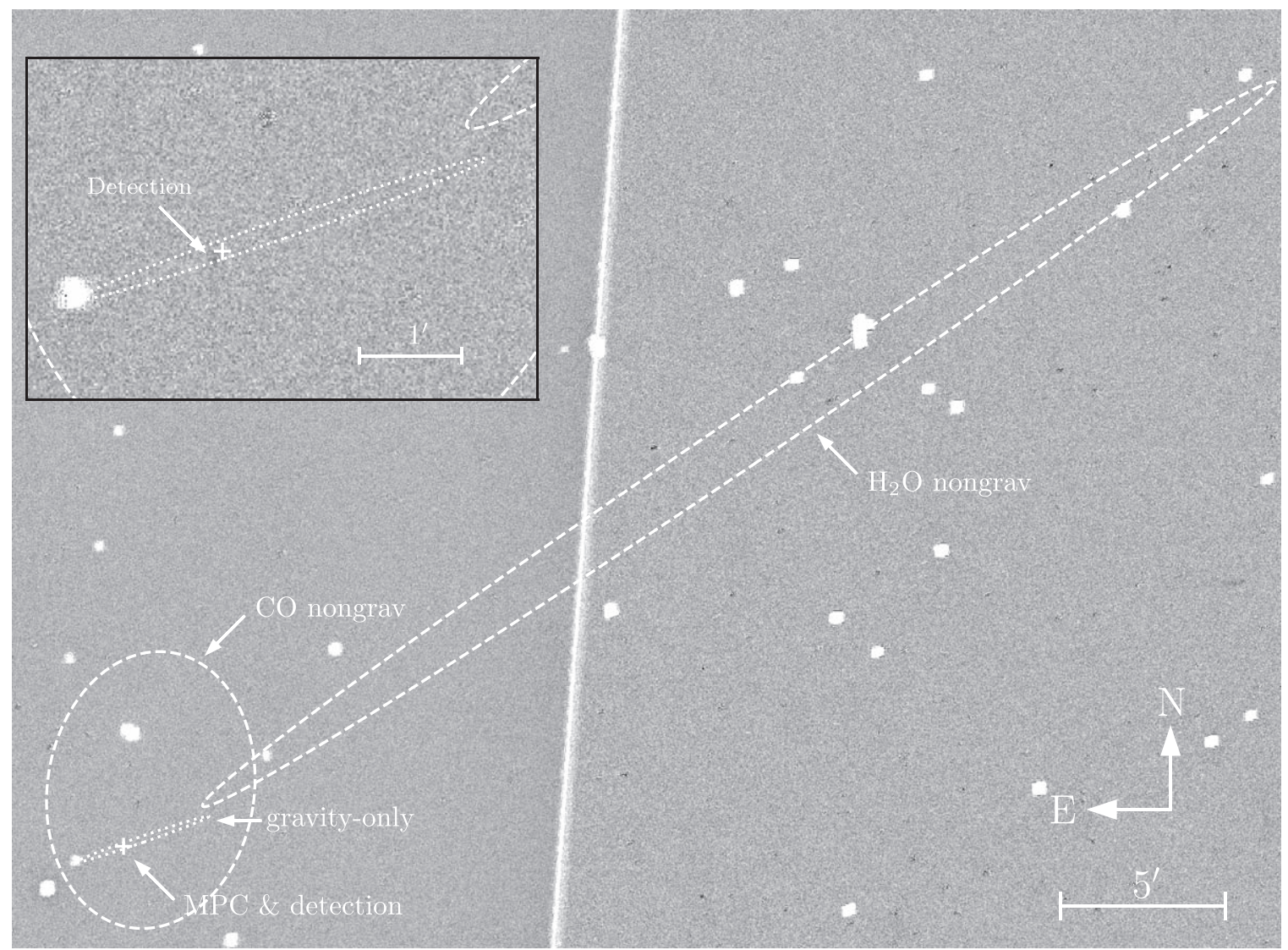

Figure 1. Combined image stacked following the predicted motion of 2I, using eight images taken on 2019 May 2, with the pre-discovery detection of the comet marked. The MPC position is calculated using the orbit published in MPEC 2019-T44. The motion rate difference between different orbit solutions is smaller than $1 / 5$ of a pixel over the entire imaging session and is negligible. The input images have been subtracted with the reference images to remove background stars (i.e., "differenced" images, see Masci et al. 2019). The white blobs are masked stars. The image is plotted in inverted linear scale (i.e., sky is white, the comet is dark).

along the major axis, and the motion rate difference between different orbit solutions is less than one-fourth of a pixel over the entire time span, but no comet is detected in the stack (Figure 3). A subsequent light-curve analysis (see Section 4.1) shows that 2I should be $\sim 1$ mag above than the $3 \sigma$ limit of the stack.

\subsection{Pan-STARRS}

The Pan-STARRS survey (Chambers et al. 2016) is a widefield asteroid survey comprised of two identical $1.8 \mathrm{~m}$ telescopes, the Pan-STARRS1 (PS1) and Pan-STARRS2 (PS2). The survey has an image archive extending back to 2010. Using the ZTF precovery data along with the available astrometry from the Minor Planet Center up to 2019 October 1, we generate an ephemeris covering the period from 2018 January 1 (when 2I was well below any practical ground-based telescope sensitivity) until the discovery date of 2019 August 30. Four $45 \mathrm{~s} i$-band images taken by the PS1 system on 2019 January 17 were identified, as summarized in Table 2. The normal processing for Pan-STARRS applies masking to remove areas not optimal for photometry because of nonuniform charge transfer efficiency, and for this study, the exposures were reprocessed without this mask. They were then visually inspected carefully over a large region centered at the expected ephemeris. The predicted ephemeris and uncertainty, however, place 2I firmly into a 70"-wide chip gap (Figure 4).

While no detections were found in the Pan-STARRS prediscovery images, the fact that the expected location of $2 \mathrm{I}$ is contained within a chip gap favors the ZTF precovery positions being correct, as even a weakly active comet should have been visible otherwise given the $G \sim 23$ limiting magnitude and near arcsecond seeing. The chip gap only occupies a small fraction $(\sim 10 \%$ based on the astrometry up to 2019 October 1$)$ of the uncertainty ellipse, therefore there was a much larger chance of being proven wrong.

However, we note that PS1 was not operational between 2018 August 23 and December 12 due to a dome shutter failure, and between 2019 February 10 and March 27 due to loss of power after a winter storm on Haleakala where the telescope is installed.

\subsection{Catalina Sky Survey (CSS)}

The CSS (e.g., Christensen et al. 2018) operates three telescopes dedicated to the discovery and follow-up of NEOs: the $0.7 \mathrm{~m}$ Catalina Schmidt at Mt. Bigelow, and $1.0 \mathrm{~m}$ and $1.5 \mathrm{~m}$ telescopes at Mt. Lemmon, Arizona.

Following a strategy similar to that for the Pan-STARRS data search, we searched the archival data of all three telescopes dating back to 2018 January 1. We identified various sets of images covering the predicted ephemeris of $2 \mathrm{I}$ : multiple sets of images taken by the $0.7 \mathrm{~m}$ Catalina Schmidt, but all dated no later than 2018 December 15, and a set of four images taken by the $1.5 \mathrm{~m}$ telescope on 2019 March 1 .

The comet was too faint ( $\gtrsim 1.5 \mathrm{mag}$ beyond the limit of the image) for the 2018 December and earlier images, while the 2019 March images reached a limiting magnitude that could be 

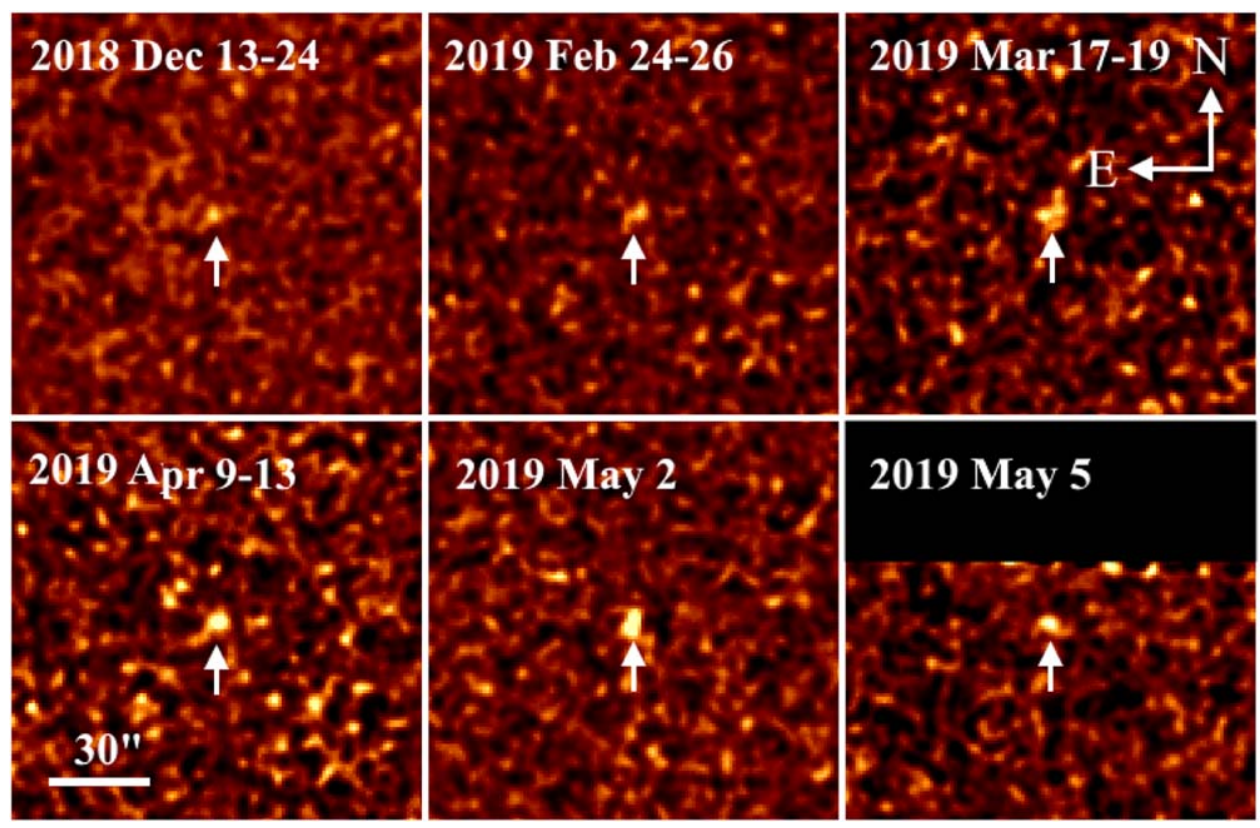

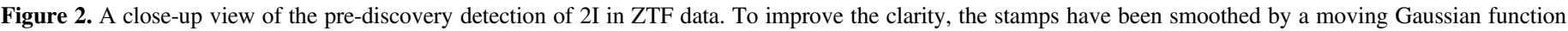
with a width of 7 pix at $1.5 \sigma$. The stamps are plotted in hyperbolic sine scale.

compatible with a faint detection of 2I. However, the position corresponding to the ZTF detections fell on a region heavily contaminated with background field sources. In three of the four frames, the object would have overlapped the pointsource-function (PSF) of a field star. The remaining frame was also marginally affected by an even brighter nearby star, but it might show a slight enhancement that is within 1.5-2 pixels from the predicted position of 2I (Figure 5). A deeper stack of historical images obtained by Catalina with the same telescope reveals no background source at that position, down to a limiting magnitude much fainter than the individual frame. Unfortunately, the enhancement was extremely faint, and might be compatible with a noise feature enhanced by the tails of the bright star's PSF, making it difficult to draw a solid conclusion.

\section{Photometric Analysis}

To measure the flux from 2I for each epoch specified in the second column of Table 2, we scale each frame in the time range listed in the first column of the table with respect to a "reference" frame in this range using the following formula for frame combination:

$$
S=10^{-0.4 \operatorname{mag}_{\mathrm{ZP}, \mathrm{corr}}}\left(\frac{\Delta}{\Delta_{0}}\right)^{2}\left(\frac{r_{\mathrm{H}}}{r_{\mathrm{H}, 0}}\right)^{4},
$$

where $S$ is the scale coefficient; $\Delta$ and $\Delta_{0}$ is the geocentric distance of the comet in the given frame and the reference frame, respectively; $r_{\mathrm{H}}$ and $r_{\mathrm{H}, 0}$ is the heliocentric distance of the comet in the given frame and the reference frame, respectively ${ }^{15}$ and the corrected magnitude zero-point

\footnotetext{
15 The exponent terms of $\Delta$ and $r_{\mathrm{H}}$ come from the classic comet brightness formula, $m_{1}=M_{1}+5 \log \Delta+10 \log r_{\mathrm{H}}$, where $m_{1}$ and $M_{1}$ are the apparent and absolute total magnitude of the comet, and the flux of the comet is proportional to $\Delta^{-2}$ and $r_{\mathrm{H}}^{-4}$, respectively. If we leave out these two terms, the non-detection photometry will differ by $0.5 \mathrm{mag}$ compared to the values listed in Table 2. For other pre-discovery data points, the differences are within $0.05 \mathrm{mag}$.
}

$\operatorname{mag}_{\text {ZP,corr }}$ is defined by

$$
\operatorname{mag}_{\mathrm{ZP}, \text { corr }}=\operatorname{mag}_{\mathrm{ZP}}+C_{\mathrm{img}} \times C_{\text {comet }},
$$

where $\operatorname{mag}_{\mathrm{ZP}}$ is the magnitude zero-point and $C_{\mathrm{img}}$ is the color coefficient derived by the ZTF Science Data System and calibrated to the PS1 photometric system (Masci et al. 2019), and $C_{\text {comet }}$ is the color of the comet. We use $C_{\text {comet: } g-r}=g_{\mathrm{PS} 1}-$ $r_{\mathrm{PS} 1}=0.54$ as measured by Guzik et al. (2019), with the colors converted using the relations derived in Tonry et al. (2012). We use a fixed photometric aperture with a radius of 5 pixels, or $21,000-29,000 \mathrm{~km}$ at the comet in the interval of 2018 November and 2019 May. This aperture is sufficient to include all flux from the comet in the pre-discovery data. The result is tabulated in Table 2.

We then fit the light curve using the classic comet light-curve equation:

$$
m_{1}=M_{1}+5 \log \Delta+K_{1} \log r_{\mathrm{H}}+\Phi(\alpha)
$$

where $m_{1}$ and $M_{1}$ are the apparent and absolute total magnitude of the comet, respectively, $K_{1}$ is the logarithmic heliocentric distance slope, and $\Phi(\alpha)$ is the phase function of the comet with respect to the phase angle $\alpha$. We note that this process should not be confused with the image scaling process with Equation (1) as described above, as the purpose of the image scaling process was to scale a subset of data to a reference epoch (defined in the 2nd column of Table 2), while the photometry of each reference epoch is then used for the lightcurve fitting described here.

We test four phase functions: the Marcus (2007) model on Halley's Comet, as well as linear phase functions $\Phi(\alpha)=\alpha \beta$ where $\beta=0.02,0.04$, and $0.06 \mathrm{mag} \mathrm{deg}^{-1}$ is the phase coefficient of the comet (Lamy et al. 2004). All four models yield comparable results, with $M_{1}$ varying from $11.6 \pm 0.1$ to $12.7 \pm 0.1$, and $K_{1}$ from $4.8 \pm 0.2$ to $5.8 \pm 0.2$, as shown in Figure 6. 
Table 2

Summary of All the Pre-discovery Observations

\begin{tabular}{|c|c|c|c|c|c|c|c|c|}
\hline Images date & Median date (UT) & Survey & $\overline{r_{\mathrm{H}}(\mathrm{au})}$ & $\Delta(\mathrm{au})$ & Images used & FWHM & Res. & $r_{\mathrm{PS} 1} \mathrm{mag}$ \\
\hline 2018 Oct $31-2018$ Nov 21 & 2018 Nov 8.82 & ZTF & 8.55 & 7.90 & $g: 12 ; r: 16$ & $1 " .4-3 " .0$ & $\cdots$ & $m_{3 \sigma}>22.69$ \\
\hline 2018 Dec 13-2018 Dec 22 & 2018 Dec 19.15 & ZTF & 7.75 & 6.99 & $g: 6 ; r: 6$ & $1 ! " 9-2 ! .7$ & 0.7 & $21.19 \pm 0.15$ \\
\hline 2019 Jan 17 & 2019 Jan 17.30 & PS1 & 7.18 & 6.58 & $i: 4$ & $0 . " 9-1 . ! 1$ & $\cdots$ & $\cdots$ \\
\hline 2019 Feb 24-2019 Feb 26 & 2019 Feb 25.18 & ZTF & 6.42 & 6.26 & $g: 3 ; r: 4$ & $1 ! \cdot 6-3 " .6$ & $0 . " 6$ & $20.97 \pm 0.18$ \\
\hline 2019 Mar 1 & 2019 Mar. 1.10 & CSS & 6.35 & 6.24 & Clear: 4 & $\sim 3^{\prime \prime}$ & $1 "$. 1 & $\gtrsim 21$ \\
\hline 2019 Mar 16-2019 Mar 18 & 2019 Mar 17.18 & ZTF & 6.02 & 6.14 & $g: 4 ; r: 5$ & $2 " .1-2 . ! 9$ & $1 . " 5$ & $20.46 \pm 0.16$ \\
\hline 2019 Apr 9-2019 Apr 13 & 2019 Apr 12.16 & ZTF & 5.53 & 5.97 & $g: 3 ; r: 3$ & $1 " 6-3 " .0$ & 1 ". 4 & $20.42 \pm 0.09$ \\
\hline 2019 May 2 & 2019 May 2.16 & ZTF & 5.15 & 5.79 & $r: 8$ & $2 !{ }^{\prime \prime} 0-2 ! ! 1$ & $1 ! ! 1$ & $20.12 \pm 0.11$ \\
\hline 2019 May 5 & 2019 May 5.15 & ZTF & 5.09 & 5.76 & $r: 4$ & $1 " .8-2 . .5$ & 0.7 & $20.16 \pm 0.21$ \\
\hline
\end{tabular}

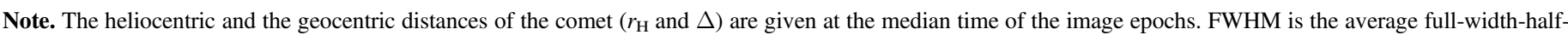
maximum of the image.

\section{Discussion}

\subsection{Driver of the Activity}

The best-fit light curve as shown in Figure 6 revealed a shallow slope, $K_{1}=5.3 \pm 0.2$, taking a linear phase coefficient $\beta=0.04 \mathrm{mag} \mathrm{deg}^{-1}$. A shallow slope means that the activity stays largely constant as the comet approaches the Sun. Whipple (1978) shows that shallow brightening is common on the "dynamically new" solar system comets (with orbital periods $\mathrm{P} \gg 10^{4} \mathrm{yr}$ ), which have $K_{1} \in(5,8)$ ), but uncommon on short-period comets and other long-period comets, which have $K_{1} \gtrsim 10$, though his data set is dominated by small heliocentric distances with $r_{\mathrm{H}}<5$ au. In this respect, $2 \mathrm{I}$ is analogous to dynamically new comets in the solar system.

The $K_{1}=5.3$ slope seemingly deviates from the data beyond $\sim 8 \mathrm{au}$, preceded by what appears like a steep brightening phase, with $K_{1} \approx 29$, though additional prediscovery observations are needed to verify it. If such a steep phase is real, it might indicate the onset of sublimation of cometary volatiles. The most compatible major volatile would be $\mathrm{CO}_{2}$, which has an onset "knee" distance $r_{\mathrm{H}}=13 \mathrm{au}$. Other cometary species, such as $\mathrm{CH}_{3} \mathrm{CN}, \mathrm{HCN}$, and $\mathrm{CH}_{3} \mathrm{OH}$, have similar turn-on distances (Meech \& Svoren 2004), but they have low abundances in solar system comets (Cochran et al. 2015). CO, another cometary volatile commonly found on dynamically new comets in solar system that has an onset distance $r_{\mathrm{H}}=120 \mathrm{au}$, is not as compatible. However, it has been suggested for the case of 'Oumuamua that the volatiles may be buried beneath the surface and are only activated when the comet is much closer to the Sun, due to the time lag for the heat wave to penetrate to the depth of the ice (Fitzsimmons et al. 2018; Seligman \& Laughlin 2018). Therefore, it is too early to exclude $\mathrm{CO}$ as the main driver of 2I's activity.

An alternative explanation of the activity is the exothermic crystallization of amorphous water ice, a mechanism that may be responsible for the activity of distant comets (Prialnik \& Bar-Nun 1990). Amorphous ice forms below an environmental temperature of $\sim 130 \mathrm{~K}$ and is capable of trapping gas as they form, a phenomenon that has been observed in laboratory experiments (Bar-Nun et al. 1987; Jenniskens \& Blake 1994), though the presence of amorphous ice is yet to be directly observed on comets. Depending on the illumination of the cometary nucleus, crystallization of amorphous ice on the surface can start around 6-12 au (Jewitt et al. 2017), consistent with the observed turn-on distance of $2 \mathrm{I}$.

To gain deeper insight into the light curve, we tested a sublimation model (Meech \& Svoren 2004) that computes the amount of gas sublimating from an icy surface exposed to solar heating to explore the activity. The total brightness within a fixed aperture combines radiation scattered from both the nucleus and the dust dragged from the nucleus in the escaping gas flow, assuming a dust-to-gas mass ratio of 1 . We used a nucleus radius of $0.5 \mathrm{~km}$ (Jewitt \& Luu 2019), assuming an albedo of 0.04 for the nucleus and a linear phase function of $0.04 \mathrm{mag} \mathrm{deg}^{-1}$ for the nucleus and $0.02 \mathrm{mag} \mathrm{deg}^{-1}$ for the coma typical of other comets (Meech \& Jewitt 1987; Krasnopolsky et al. 1987), a nucleus density of $400 \mathrm{~kg} \mathrm{~m}^{-3}$ similar to that seen for comets 9P/Tempel 1, 103P/Hartley 2 (Thomas 2009), and 67P/Churyumov-Gerasimenko (Pätzold et al. 2016), a grain density of $800 \mathrm{~kg} \mathrm{~m}^{-3}$ (Fulle et al. 2016), and large $(10-100 \mu \mathrm{m})$ grains (Fitzsimmons et al. 2019). Unsurprisingly, our model confirmed that the activity of 2I must be driven by a species more volatile than water, otherwise it would have been well below the detection limit of any of the surveys at $\sim 5$ au. We also found that the differences between the shape of the sublimation curves for $\mathrm{CO}$ and $\mathrm{CO}_{2}$ near $8 \mathrm{au}$ is minimal, so it is impossible to distinguish between these volatiles without further pre-discovery observations.

\subsection{Size of the Nucleus}

The non-detection in 2018 November data can be used to constrain the size of the nucleus. The effective cross-section area for scattering can be calculated using

$$
C_{\mathrm{e}}=\frac{\pi(1 \mathrm{au})^{2}}{p} 10^{-0.4\left(M_{r}-m_{\odot, r}\right)},
$$

where $p=0.04$ is the assumed optical geometric albedo of the nucleus (Lamy et al. 2004), $m_{\odot, r}=-26.9$ is the apparent $r$-band magnitude of the Sun (Willmer 2018), and the absolute $r$-band magnitude (technically, the upper limit) $M_{r}$ is defined as

$$
M_{r}=m_{r}-5 \log r_{\mathrm{H}} \Delta-\Phi \alpha,
$$

with the variables following the same definitions for Equation (3). By inserting all the numbers, we have $C_{\mathrm{e}}<$ $140 \mathrm{~km}^{2}$ for the non-detection in 2018 November, at $8.5 \mathrm{au}$. This upper bound indicates that the radius of the nucleus is no larger than $\sim 7 \mathrm{~km}$.

\subsection{Active Area on the Nucleus}

The size of the active area on the nucleus can be estimated with knowledge of the mass-loss rate of the comet and the mass flux of the activity-driving volatiles. The mass-loss rate of 2I 


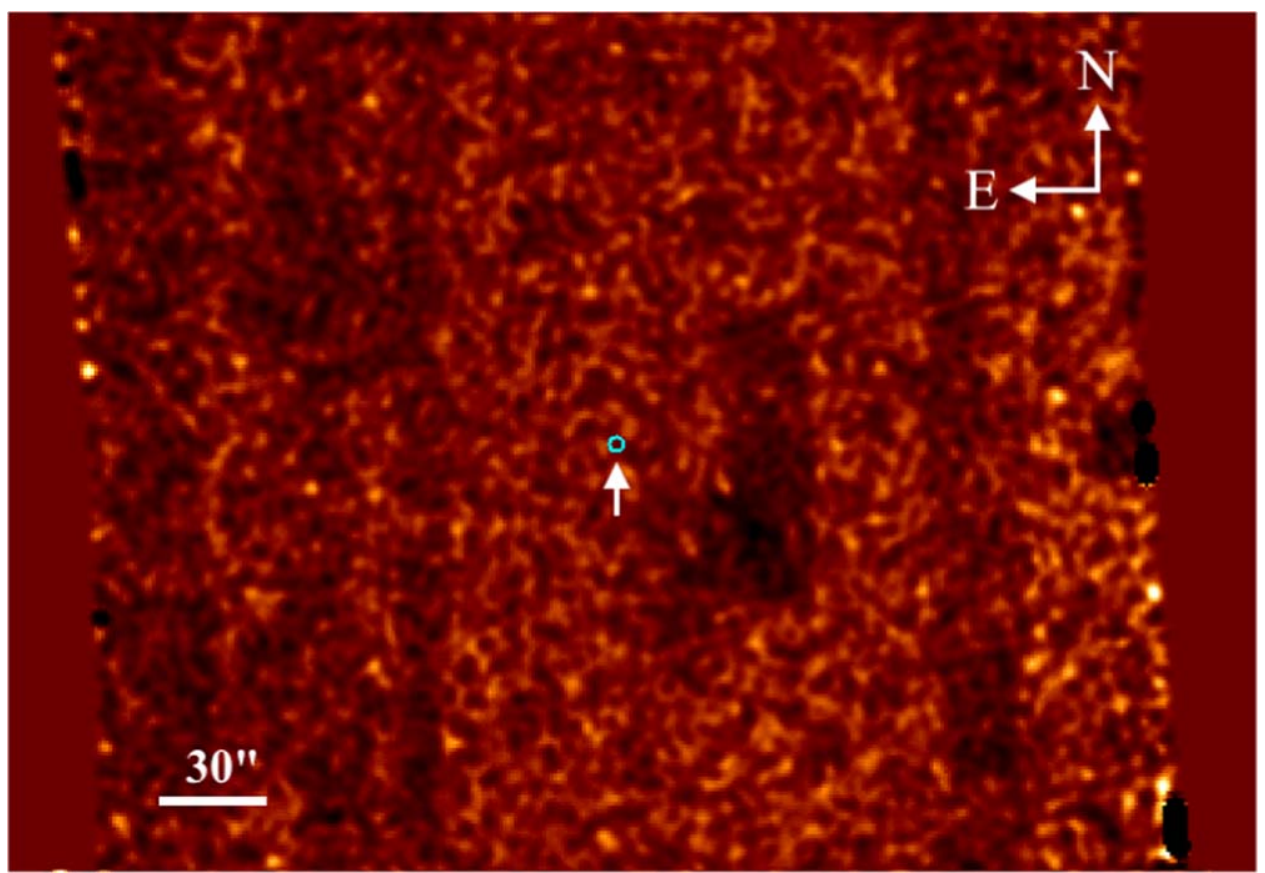

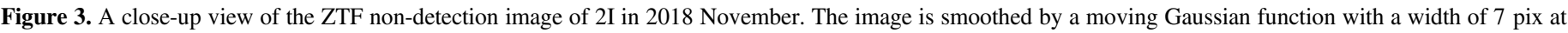
$1.5 \sigma$ and is plotted in hyperbolic sine scale. The circle in cyan marks the nominal of the comet and the size of the $3 \sigma$ uncertainty.

can be estimated using the cross-section area of the dust and the speed of the dust flow. Using Equation (4), we derive the crosssection area of the coma to be $\sim 280-360 \mathrm{~km}^{2}$, from March to October 2019, in which $r_{\mathrm{H}}$ decreases from 6.4 to $2.6 \mathrm{au}$. The mass-loss rate $\dot{M}$ can be calculated by

$$
\dot{M}=\frac{4 \rho \bar{a} C_{\mathrm{e}}}{3 \tau},
$$

where $\rho=800 \mathrm{~kg} \mathrm{~m}^{-3}$ is the bulk density of the dust (Fulle et al. 2016), which is admittedly not yet constrained for interstellar comets, but we do not have reason to believe it is much different from solar system comets, and therefore have assumed it to be analogous to the latter, $\bar{a}=10^{-4} \mathrm{~m}$, the characteristic size of the dust, which is similarly assumed based on the observation of dynamically new solar system comets (e.g., Ye \& Hui 2014; Jewitt et al. 2019), and $\tau$ is the timescale on which a dust particle moves out of the aperture, which can be estimated by $\tau=l / v \approx 10^{5} \mathrm{~s}$, where $l$ is the linear length of the aperture at the comet, and $v \sim 1 \mathrm{~m} \mathrm{~s}^{-1}$ is the ejection speed of the dust, taking the dust speed constrained by Guzik et al. (2019) and assuming a classic $\propto a^{-0.5}$ dependence. By inserting all the numbers, we obtain $\dot{M} \approx 10 \mathrm{~kg} \mathrm{~s}^{-1}$, with the uncertainty around an order of magnitude mainly owing to the parameter $\bar{a}$ (which can vary by an order of magnitude among solar system comets; see Fulle 2004).

We then solve the energy balance equation for $\mathrm{CO}$ and $\mathrm{CO}_{2}$ ice, which are likely to be responsible for 2I's activity following the discussion in Section 4.1, at the sub-solar point on the nucleus:

$$
\frac{\left(1-A_{B}\right) L \odot}{4 \pi r_{\mathrm{H}}^{2}}=\epsilon \sigma T^{4}+Z(T) L(T),
$$

where $A_{B}=0.01$ is the Bond albedo of the nucleus measured for 9P/Tempel 1 and 103P/Hartley 2 (Li et al. 2013a, 2013b), $L_{\odot}$ is the luminosity of the Sun, $\epsilon=0.9$ is the infrared emissivity of the nucleus, $\sigma$ is the Boltzmann constant, $L(T)$ is the latent heat of the sublimation of the ice, and $Z(T)$ is the mass flux. We solve $L(T)$ using the model by Cowan \& A'Hearn (1979), ${ }^{16}$ and obtain $Z(T) \approx 3 \times 10^{-6}-2 \times 10^{-5} \mathrm{~kg} \mathrm{~m}^{-2} \mathrm{~s}^{-1}$ from 6.4 to 2.6 au for $\mathrm{CO}$, and $1 \times 10^{-6}-9 \times 10^{-6} \mathrm{~kg} \mathrm{~m}^{-2} \mathrm{~s}^{-1}$ for the same $r_{\mathrm{H}}$ span for $\mathrm{CO}_{2}$. The active area required to support the mass-loss rate would then be

$$
A=\frac{\dot{M}}{\left(M_{\mathrm{d}} / M_{\mathrm{g}}\right) Z(T)},
$$

where $M_{\mathrm{d}} / M_{\mathrm{g}}$ is the dust-to-gas mass ratio, which is again unknown for interstellar comets. If we take $M_{\mathrm{d}} / M_{\mathrm{g}}=1$ based on the measurement of long-period comet $\mathrm{C} / 1995$ O1 (Hale-Bopp) at similar heliocentric distance (Weiler et al. 2003), we have $A=0.5-10 \mathrm{~km}^{2}$ over the time span between 2018 December and 2019 September, in line with the number derived by McKay et al. (2020) based on their observation in 2019 October $\left(1.7 \mathrm{~km}^{2}\right)$. Taking the upper limit of the size of the nucleus derived in Section 4.2, the active fraction of $2 \mathrm{I}$ is $>0.1 \%$ of the nucleus. This is in line with the known solar system comets, which have fractional active areas from a few $0.1 \%$ to $>100 \%$ (Tancredi et al. 2006), though we note that most of these measured comets are shortperiod comets with $\mathrm{H}_{2} \mathrm{O}$ as the driving species. However, we caution that the uncertainty in $A$ is about 1-2 orders of magnitude when we consider the uncertainties in $\dot{M}$ and $M_{\mathrm{d}} / M_{\mathrm{g}}$, therefore the derived $A$ and active fraction are highly uncertain.

\footnotetext{
${ }^{16}$ https://pdssbn.astro.umd.edu/SBNcgi/newiso.cgi
} 


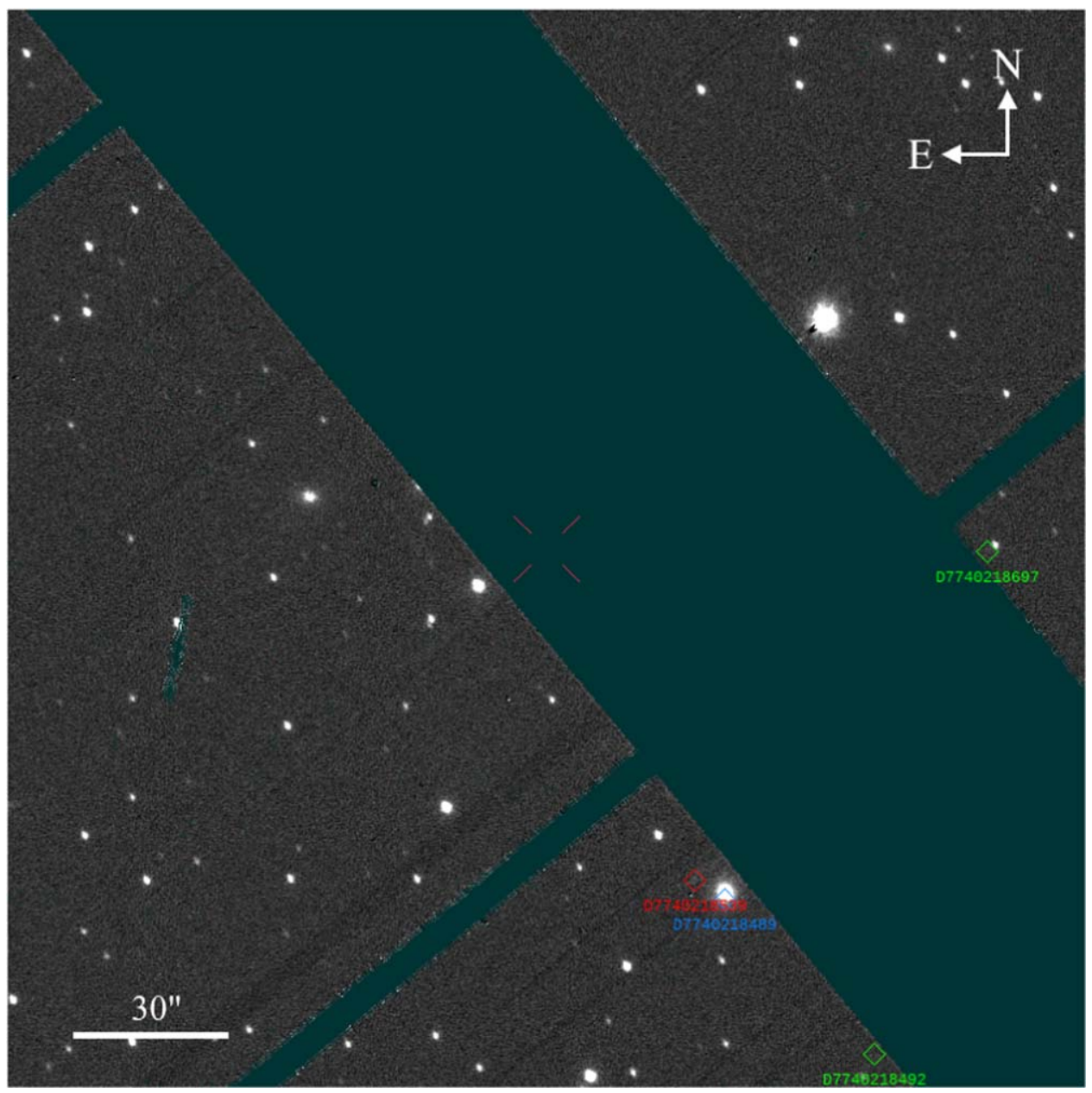

Figure 4. Precovery image of 2I taken by PS1 on 2019 January 17. The comet's nominal location, marked by a red crosshair, is in the chip gap.

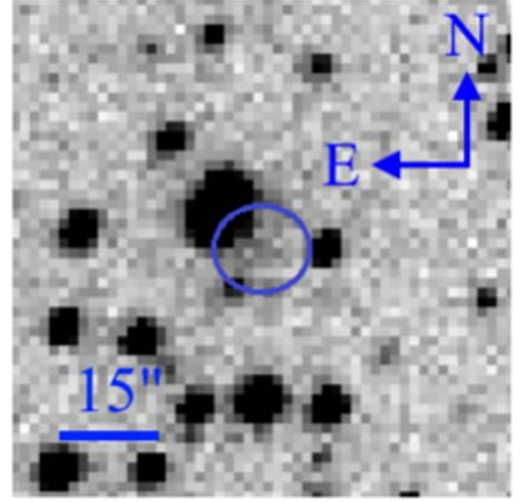

Figure 5. Precovery image of 2I taken by the CSS $1.5 \mathrm{~m}$ telescope on 2019 March 1 . The slight enhancement possibly corresponds to the comet is marked by a blue circle. The color is inverted.

\subsection{Non-gravitational Accelerations and Implications}

The inclusion of the precovery data in the orbit estimation process provides more stringent constraints on the trajectory of 2I, but also introduces challenges to correctly modeling the dynamics. A gravity-only model of the orbit struggles to fit the data of 2019 March and earlier. In particular, the 2018 December position is rejected as an outlier (using the outlier rejection algorithm by Carpino et al. 2003). At this stage it is not entirely clear whether this behavior is caused by systematic errors in the bulk of the astrometric data, which were taken at low solar elongation and therefore at high airmass, or by nongravitational accelerations. We note that non-gravitational accelerations were detected in the motion of 'Oumuamua, despite the lack of visible outgassing (Micheli et al. 2018).

Table 1 reports JPL solution 37, which fits all the precovery observations and uses non-gravitational forces assuming $\mathrm{CO}$ as the primary driver (Section 2.1), more consistent than $\mathrm{H}_{2} \mathrm{O}$ with the photometric data. The non-gravitational model for $\mathrm{CO}_{2}$ is not available at this point; but since both $\mathrm{CO}$ and $\mathrm{CO}_{2}$ are more volatile than water and 2I was in the regime of both volatiles when it was observed, we believe that the two models should behave similarly over the fit span.

We also tested the rotation jet model (Chesley \& Yeomans 2005), which computes the non-gravitational perturbations from a discrete number of jets, whose acceleration is averaged over a nucleus rotation. For the driver of the activity we again used $\mathrm{CO}$. We considered two jets, a nearly polar one at $10^{\circ}$ of colatitude and a midlatitude one on the southern hemisphere at $135^{\circ}$ of colatitude. Then, we scanned a raster for the spin pole's R.A. and decl., estimating the strengths of the two jets from the fit to the astrometry.

As shown in Figure 7, we find two minima for the pole's R.A. and decl.: $\left(340^{\circ},+30^{\circ}\right)$ and $\left(205^{\circ},-55^{\circ}\right)$. Because of the larger number of parameters, the jet model provides a better fit to the data than the Marsden et al. (1973) model. However, its reliability will need to be validated by its ability to make 


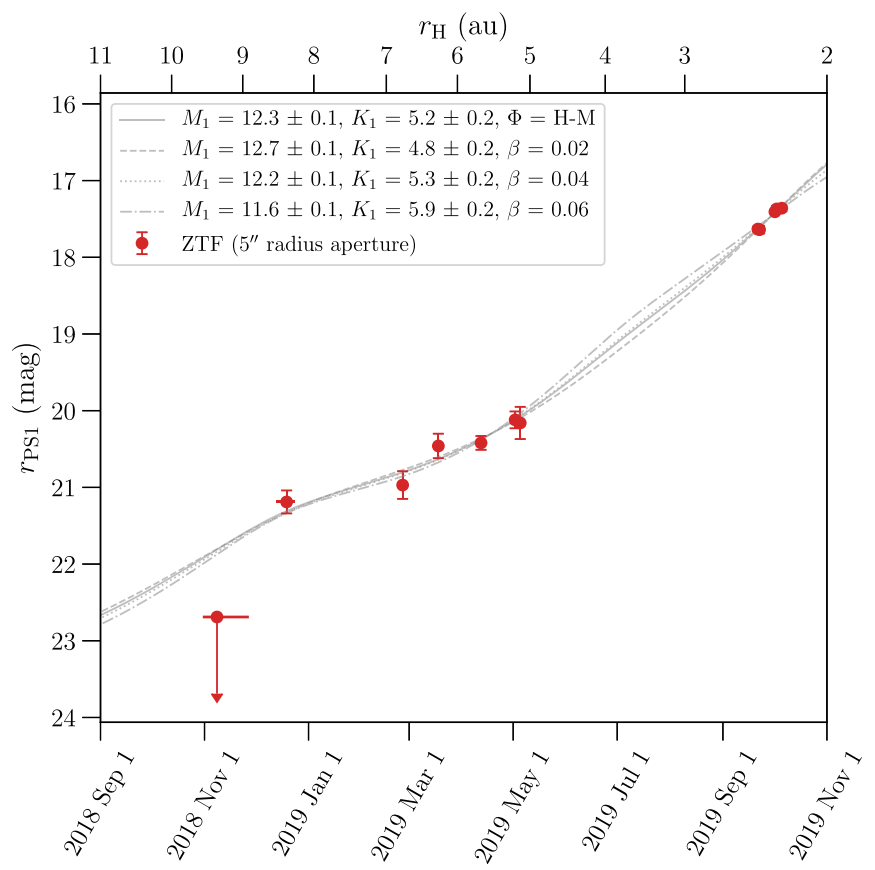

Figure 6. Best-fit light curve of $2 \mathrm{I}$ using the pre-discovery photometry and recent ZTF photometry (MPEC 2019-V34 and MPEC 2019-W50). Horizontal bars indicate the time bin sizes. The light-curve functions being fitted are the Marcus model on Halley's Comet $(\mathrm{H}-\mathrm{M})$, as well as linear phase functions assuming a phase coefficient of $0.02,0.04$, and $0.06 \mathrm{mag} \mathrm{deg}^{-}$. The bumps in 2018 December and 2019 July are caused by brightness enhancements at small phase angles during the opposition and conjunction of 2I.

accurate predictions. Past experience has shown that the jet model can provide more accurate comet trajectory estimates (Farnocchia et al. 2016) and therefore the jet model solutions are worth consideration, especially as the observed arc extends into late 2019 and 2020.

\section{Conclusions}

The pre-discovery observations of newly-discovered interstellar comet 2I/Borisov revealed a comet that is observationally quite comparable to the long-period dynamically new comets in our own solar system. We found that 2I was active at 5-7 au, indicative of the presence of accessible ices more volatile than $\mathrm{H}_{2} \mathrm{O}$, such as $\mathrm{CO}$ and $\mathrm{CO}_{2}$. A subsequent comprehensive follow-up campaign, presented by Bolin et al. (2019), reinforces this conclusion. We identified a possible steep brightening at 8-9 au that might indicate an onset of activity at this distance, which suggests crystallization of amorphous ice as an alternative mechanism for the activity, but more pre-discovery data, preferably from larger, multi-metersized telescopes, is needed to verify this behavior. We also found the nucleus to be no more than $7 \mathrm{~km}$ in radius, and that $\gtrsim 0.1 \%$ of the surface is currently active, both are quite typical when compared to dynamically new solar system comets occasionally discovered and observed by surveys, though the derived size of active area is highly uncertain, mainly due to the uncertainties in nucleus size and dust size distribution. The prediscovery observations also provide stronger constraints on the inbound trajectory and non-gravitational forces of 2I. We found that a $\mathrm{CO}$ model provides results that are more consistent with the observations compared to the $\mathrm{H}_{2} \mathrm{O}$ model.

It will be interesting to see if $2 \mathrm{I}$ continues to fit into the profile of dynamically new comets. For solar system comets, it

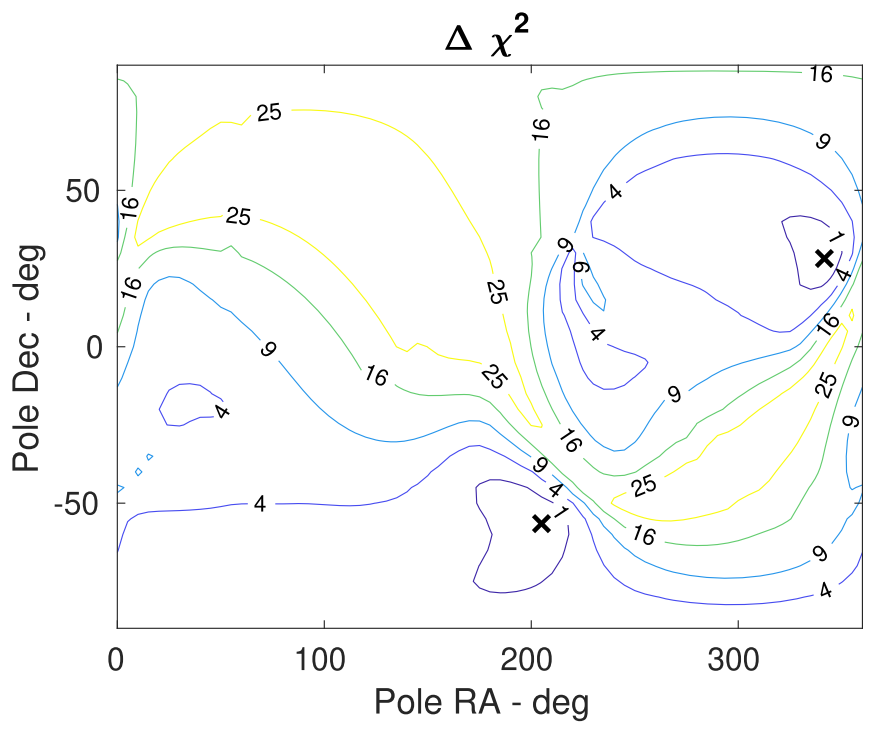

Figure 7. $\Delta \chi^{2}$ of the astrometric fit for the jet model as a function of the pole orientation. The two minima are marked with a black cross.

is known that dynamically new comets are $10 \times$ more likely to disintegrate than short-period comets, presumably due to their pristine state and weaker structural strength (Weissman et al. 2004). We note that an independent analysis by Jewitt \& Luu (2019) also suggested that 2I may be prone to disintegration based on its small nucleus size (sub-kilometer-sized). Comets can disintegrate at large heliocentric distances, but most disintegrations seem to happen within $\sim 2$ au (Boehnhardt 2004), a distance that 2I will reach at its perihelion in December 2019. Surviving dynamically new comets also tend to fade more rapidly after perihelion (Whipple 1978). Continued observations of 2I will enable further comparison to dynamically new comets in our solar system, and provide timely warning for any disintegration (or, less dramatically, outburst) that may happen.

The authors thank Man-To Hui and David Jewitt for discussions of the CO model, as well as George Helou, Matthew Knight, Zhong-Yi Lin, Ralph Roncoli, Qicheng Zhang, and the anonymous referee for comments. M.S.P.K. acknowledges support from NASA grant NNX17AK15G. D.F. conducted this research at the Jet Propulsion Laboratory, California Institute of Technology, under a contract with NASA.

This work is based on observations obtained with the Samuel Oschin 48-inch Telescope at the Palomar Observatory as part of the Zwicky Transient Facility project. ZTF is supported by the National Science Foundation under grant No. AST1440341 and a collaboration including Caltech, IPAC, the Weizmann Institute for Science, the Oskar Klein Center at Stockholm University, the University of Maryland, the University of Washington, Deutsches Elektronen-Synchrotron and Humboldt University, Los Alamos National Laboratories, the TANGO Consortium of Taiwan, the University of Wisconsin at Milwaukee, and Lawrence Berkeley National Laboratories. Operations are conducted by Caltech Optical Observatories, the Infrared Processing and Analysis Center, and the University of Washington.

Pan-STARRS is supported by the National Aeronautics and Space Administration under grant No. 80NSSC18K0971 
issued through the SSO Near Earth Object Observations Program.

Facilities: PO:1.2 m, PS1, SO:1.5m.

Software: ZChecker (Kelley et al. 2019).

\section{ORCID iDs}

Quanzhi Ye (叶泉志) (1) https://orcid.org/0000-00024838-7676

Michael S. P. Kelley (1) https://orcid.org/0000-00026702-7676

Dennis Bodewits (1) https://orcid.org/0000-0002-2668-7248

Davide Farnocchia (1) https://orcid.org/0000-0003-0774-884X

Frank J. Masci (1) https://orcid.org/0000-0002-8532-9395

Karen J. Meech iㅏ https://orcid.org/0000-0002-2058-5670

Marco Micheli iㅏ https://orcid.org/0000-0001-7895-8209

Robert Weryk iㅏ https://orcid.org/0000-0002-0439-9341

Eric C. Bellm (ib https://orcid.org/0000-0001-8018-5348

Matthew J. Graham (1) https://orcid.org/0000-0002-3168-0139

Shrinivas R. Kulkarni (ib https://orcid.org/0000-0001-

5390-8563

Russ R. Laher (1) https://orcid.org/0000-0003-2451-5482

Ben Rusholme (1) https://orcid.org/0000-0001-7648-4142

\section{References}

Bar-Nun, A., Dror, J., Kochavi, E., \& Laufer, D. 1987, PhRvB, 35, 2427 Bellm, E. C., Kulkarni, S. R., Graham, M. J., et al. 2019, PASP, 131, 018002 Biver, N., Rauer, H., Despois, D., et al. 1996, Natur, 380, 137

Boehnhardt, H. 2004, in Comets II, ed. M. C. Festou, H. U. Keller, \& H. A. Weaver (Tucson, AZ: Univ. Arizona Press), 301

Bolin, B. T., Lisse, C. M., Kasliwal, M. M., et al. 2019, arXiv:1910.14004

Carpino, M., Milani, A., \& Chesley, S. R. 2003, Icar, 166, 248

Chambers, K. C., Magnier, E. A., Metcalfe, N., et al. 2016, arXiv: 1612.05560

Chesley, S. R., \& Yeomans, D. K. 2005, in IAU Coll. 197: Dynamics of Populations of Planetary Systems, ed. Z. Knežević \& A. Milani (Cambridge: Cambridge Univ. Press), 289

Christensen, E., Africano, B., Farneth, G., et al. 2018, AAS/DPS Meeting, 50, 310.10

Cochran, A. L., Levasseur-Regourd, A.-C., Cordiner, M., et al. 2015, SSRv, 197, 9

Cowan, J. J., \& A'Hearn, M. F. 1979, M\&P, 21, 155

de León, J., Licandro, J., Serra-Ricart, M., et al. 2019, RNAAS, 3, 131

Farnocchia, D., Chesley, S. R., Micheli, M., et al. 2016, Icar, 266, 279

Fitzsimmons, A., Hainaut, O., Meech, K., et al. 2019, ApJL, 885, L9

Fitzsimmons, A., Snodgrass, C., Rozitis, B., et al. 2018, NatAs, 2, 133

Fulle, M. 2004, in Comets II, ed. M. C. Festou, H. U. Keller, \& H. A. Weaver (Tucson, AZ: Univ. Arizona Press), 565

Fulle, M., Altobelli, N., Buratti, B., et al. 2016, MNRAS, 462, S2
Graham, M. J., Kulkarni, S. R., Bellm, E. C., et al. 2019, PASP, 131, 078001 Gunnarsson, M., Rickman, H., Festou, M., Winnberg, A., \& Tancredi, G. 2002, Icar, 157, 309

Guzik, P., Drahus, M., Rusek, K., et al. 2019, ATel, 13100, 1

Jenniskens, P., \& Blake, D. F. 1994, Sci, 265, 753

Jewitt, D., Agarwal, J., Hui, M.-T., et al. 2019, AJ, 157, 65

Jewitt, D., Hui, M.-T., Mutchler, M., et al. 2017, ApJL, 847, L19

Jewitt, D., \& Luu, J. 2019, ApJL, 886, L29

Kelley, M. S. P., Bodewits, D., Ye, Q., et al. 2019, in ASP Conf. Ser.471, Astronomical Data Analysis Software and Systems XXVIII, ed. P. J. Teuben, M. W. Pound, \& B. A. Thomas (San Francisco, CA: ASP), 471

Krasnopolsky, V. A., Moroz, V., Krysko, A., Tkachuk, A. Y., \& Moreels, G. 1987, A\&A, 187, 707

Lamy, P. L., Toth, I., Fernandez, Y. R., \& Weaver, H. A. 2004, in Comets II, ed. M. C. Festou, H. U. Keller, \& H. A. Weaver (Tucson, AZ: Univ. Arizona Press), 223

Li, J.-Y., A'Hearn, M. F., Belton, M. J. S., et al. 2013a, Icar, 222, 467

Li, J.-Y., Besse, S., A'Hearn, M. F., et al. 2013b, Icar, 222, 559

Marcus, J. N. 2007, ICQ, 29, 39

Marsden, B. G., Sekanina, Z., \& Yeomans, D. K. 1973, AJ, 78, 211

Masci, F. J., Laher, R. R., Rusholme, B., et al. 2019, PASP, 131, 018003

McKay, A. J., Cochran, A. L., Dello Russo, N., \& DiSanti, M. 2020, ApJL, 889, L10

Meech, K. J., \& Jewitt, D. C. 1987, A\&A, 187, 585

Meech, K. J., \& Svoren, J. 2004, in Comets II, ed. M. C. Festou, H. U. Keller, \& H. A. Weaver (Tucson, AZ: Univ. Arizona Press), 317

Meech, K. J., Weryk, R., Micheli, M., et al. 2017, Natur, 552, 378

Micheli, M., Farnocchia, D., Meech, K. J., et al. 2018, Natur, 559, 223

Opitom, C., Fitzsimmons, A., Jehin, E., et al. 2019, A\&A, 631, L8

Oumuamua ISSI Team, Bannister, M. T., Bhand are, A., et al. 2019, NatAs, 3, 594

Pätzold, M., Andert, T., Hahn, M., et al. 2016, Natur, 530, 63

Prialnik, D., \& Bar-Nun, A. 1990, ApJ, 363, 274

Seligman, D., \& Laughlin, G. 2018, AJ, 155, 217

Tancredi, G., Fernández, J. A., Rickman, H., \& Licandro, J. 2006, Icar, 182,527

Thomas, N. 2009, P\&SS, 57, 1106

Tonry, J. L., Stubbs, C. W., Lykke, K. R., et al. 2012, ApJ, 750, 99

Weiler, M., Rauer, H., Knollenberg, J., Jorda, L., \& Helbert, J. 2003, A\&A, 403, 313

Weissman, P. R., Asphaug, E., \& Lowry, S. C. 2004, in Comets II, ed. M. C. Festou, H. U. Keller, \& H. A. Weaver (Tucson, AZ: Univ. Arizona Press), 337

Whipple, F. L. 1978, M\&P, 18, 343

Willmer, C. N. A. 2018, ApJS, 236, 47

Yabushita, S. 1996, MNRAS, 283, 347

Ye, Q.-Z., \& Hui, M.-T. 2014, ApJ, 787, 115

Ye, Q.-Z., Masci, F. J., Ip, W.- H., et al. 2020, AJ, 159, 70

Ye, Q.-Z., Zhang, Q., Kelley, M. S. P., \& Brown, P. G. 2017, ApJL, 851, L5

Yeomans, D., Chodas, P., Sitarski, G., Szutowicz, S., \& Królikowska, M. 2004, in Comets II, ed. M. C. Festou, H. U. Keller, \& H. A. Weaver (Tucson, AZ: Univ. Arizona Press), 137 